EESTI NSV TEADUSTE AKADEEMIA TOIMETISED. XI KOIDE FUOSIKALIS-MATEMAATILISTE JA TEHNILISTE TEADUSTE SEERIA. 1962, NR. 2

ИЗВЕСТИЯ АКАДЕМИИ НАУК ЭСТОНСКОН ССР. ТОМ ХІ

СЕРИЯ ФИЗИКО-МАТЕМАТИЧЕСКНХ И ТЕХНИЧЕСКИХ НАУК. 1962, № 2

\title{
ОБ ОБОБЩЕННОЙ ТЕОРЕМЕ МЕРСЕРА
}

\section{T. СЫРМУС}

В статье будет установлена связь обобщенной теоремы Мерсера, доказанной Питтом и Петерхаусом [3], с одной из рассмотренных нами ранее теорем (2.1 из []) путем доказательства теоремы, которая при определенных условиях заменяет теорему Питта и Петерхауса. Далее докажем аналоги обобщенных теорем-Mерсера для двойных последовательностей, ограничиваясь при этом факторизирующимися методами Хаусдорфа.

В статье мы пользуемся символикой из предыдущей статьи [7].

1. Сначала приведем нужные нам теоремы и одну лемму.

T е орем а 1.1. (Питт и Петерхаус $\left[{ }^{3}\right]$, теорема 7.)

Пуусть $\mathrm{X}(t)$ - функция с ограниченным изменением без сингулярной составляющей и $\mathrm{X}(+0)=\mathrm{X}(0)=0, \quad \mathrm{X}(1)=1$. Для того чтобы из $\left(H, \int_{0}^{1} t^{k} d \mathrm{X}(t)\right)$-суммируемости последовательности $\left\{s_{k}\right\}$ вытекала сходимость этой последовательности, необходимо и. достаточно выполнение условия

$$
\inf _{R \omega}\left|\int_{0}^{1} t^{\omega} d \mathrm{X}(t)\right|>0
$$

Т е ор ем а 1.2. (Виланский, [4].)

Сохраняюиций сходимость обратимый метод А суммирует расходящуюся последовательность всегда только при условии

$$
\sup _{n} \sum_{k}\left|a_{n k}^{\prime}\right|=\infty
$$

Те орем а 1.3. (Адамс ['], теорема 7.)

Если $A^{\prime}$ и $A^{\prime \prime}$ обраттиме методы простых последовательностей, то метод $A=A^{\prime} \odot A^{\prime \prime}$ и.неет обратный метод вида $A^{-1}=\left(A^{\prime}\right)^{-1} \odot\left(A^{\prime \prime}\right)^{-1}$.

Определение метода $\left(H, \mu_{k}\right)=\left(H, \int_{0}^{1} t^{k} d \mathrm{X}(t)\right)$ дано в [8]. 
Tе о рем а 1.4. (Адамс ['], теорема 14.)

Если $A^{\prime}$ и $A^{\prime \prime}$ регулярные методы простых последовательностей, то метод $A=A^{\prime} \odot A^{\prime \prime}$ регулярен в том классе двойных последовательностей, A-преобразование которых ограничено.

T е ор е м 1.5. (Адамс $\left[{ }^{2}\right]$, теорема 3.)

Если метод $\left(H, \mu_{k} v_{l}\right)=\left(H, \mu_{k}\right) \odot\left(H, v_{l}\right)$ сохраняет сходимость, но не регулярен для ну.цевых последовательностей, то $\left(H, \mu_{k}\right) u\left(H, v_{l}\right)$ такюе сохраняют сходимость, но не-регулярны для нулевых последовательностей.

T ео ре м а 1.6. (Адамс [2], теорема 4.)

Если метод $\left(H, \mu_{k} v_{l}\right)=\left(H, \mu_{k}\right) \odot\left(H, v_{l}\right)$ сохраняет сходимость и регулярен для нулевых последовательностей (при условии, что не все элементы равны нуло), то $\left(H, \mu_{k}\right) u\left(H, v_{l}\right)$ сохраняют сходимость и хоть один из них регулярен для нулевых последовательностей.

T е о ре м а 1.7. (Сырмус [5], теорема 2.2.)

Tреугольный регулярный метод $A=\left(a_{m n k l}\right)$ ограниченно суммирует расходяиуюся последовательность всегда только при условии

$$
\sup _{m, n} \sum_{k, l=0}^{m, n}\left|a_{m n k l}^{\prime}\right|=\infty \text {. }
$$

Л ем м а 1.1 Если Х(t) - функция с ограниченным изменением без сингулярной состивляющей и $\mathrm{X}(+0)=\mathrm{X}(0)=0, \quad \mathrm{X}(1)=1, \inf _{R_{\omega} \geqslant 0}\left|\int_{0}^{1} t^{\omega} d \mathrm{X}(t)\right|>0$, то последовательность Хаусдорфа $\left\{\frac{1}{\mu_{k}}\right\}$, где $\mu_{k}=\int_{0}^{1} t^{k} \mathrm{X}(t)$, регулярна.

Доказательство леммы аналогично доказательству леммы в предыдущей статье (лемма 1.2 из [?]).

2. В [] доказана следующая теорема.

Те орема 2.1 Если $\lambda>0, h \neq \infty$, a последовательность Хаусдорфа $\left\{\mu_{k}\right\}$ абсолотно монотонна и регулярна, то из равенства

$$
\lim _{n}\left(\lambda s_{n}+(1-\lambda) \sum_{k=0}^{n-1}\left({ }_{k}^{n-1}\right) \Delta^{n-1-k} \mu_{k} s_{k}\right)=h
$$

вытекает

$$
\lim _{n} s_{n}=h .
$$

Для установления связи приведенной теоремы с теоремой 1.1 докажем следующую теорему.

Те о рем а 2.2. Пусть $\lambda>0, h \neq \infty u\left\{\mu_{k}\right\}$ - абсолютно монотонная последовательность Хаусдорфа, удовлетворяющая условию $\lim \Delta^{n} \mu_{0}=0$. Для того итобы из равенства

$$
\lim _{n}\left(\lambda s_{n}+(1-\lambda) \sum_{k=0}^{n-1}\left({ }^{n-1}\right) \Delta^{n-1-k} \mu_{k} s_{k}\right)=h
$$


вытекало равенство

$$
\lim _{n} s_{n}=h
$$

необходимо и достаточно, чтобы $\mu_{0}=1$.

Док а зат ельств о. Достаточность условия следует из теоремы 2.1. Докажем его необходимость.

Пусть

$$
t_{n}=\lambda s_{n}+(1-\lambda) H_{n-1} \text {, }
$$

где

$$
H_{n-1}=\sum_{k=0}^{n-1}\left({ }_{k}^{n-1}\right) \Delta^{n-1-k} \mu_{k} s_{k}
$$

Так же, как и в доказательстве одной нашей теоремы (2.1 в [6]), получаем

$$
H_{n}=\sum_{k=0}^{n} \frac{\gamma_{n k}}{\lambda \gamma_{n n} h_{n n}^{\prime}} t_{k}
$$

причем величины $\gamma_{n k}$ определены известной системой (2.3 из [']). По условию теоремы из равенства $\lim t_{n}=h$ следует $\lim s_{n}=h$, а ввиду равенства (2.1) еще и $\lim H_{n}=h$. Но тогда метод, определенный матрицей $\left(\frac{\gamma_{n k}}{\lambda \gamma_{n n} h_{n n}^{\prime}}\right)$, регулярен, т.е. удовлетворяет условию

$$
\lim _{n} \sum_{k=0}^{n} \frac{\gamma_{n k}}{\lambda \gamma_{n n} h_{n n}^{\prime}}=1
$$

Для определения величины $\sum_{k=0}^{n} \frac{\gamma_{n b}}{\lambda \gamma_{n n^{\prime}{ }_{n n}^{\prime}}}$ предполагаем в (2.2) $s_{k} \equiv 1$; тогда $H_{n} \equiv \mu_{0}$, а на основании $(2.1)$ имеем $t_{n}=\lambda+(1-\lambda) \mu_{0}$. Учитывая это, получаем из $(2.3)^{\circ}$ и $(2.4)$, что

$$
\lim _{n} \sum_{k=0}^{n} \frac{\gamma_{n k}}{\lambda \cdot v_{n n} h_{n n}^{\prime}}=\frac{\mu_{0}}{n+(1-\lambda) \mu_{0}}=1,
$$

т. е. $\mu_{0}=1$, что и требовалось доказать.

Нам необходима еще следующая

теорем а 2.3. Если $\lambda>0 u\left\{\mu_{k}\right\}$ - абсолютно монотонная последовательность Хаусдорфа, то из сходимости последовательности $t_{n}=\lambda s_{n}+(1-\lambda) \sum_{k=0}^{n-1}\left({ }_{k}^{n-1} \mathbf{y} \mu_{k} s_{k}\right.$ вытекает сходимость последовательности $\left\{s_{n}\right\}$.

Док аз а тельство теоремы аналогично доказательству теоремы 2.1 .

Теперь докажем теорему, которая является аналогом теоремы 1.1.

Т ео о ем а 2.4. Пусть $\left\{v_{k}\right\}-$ абсолютно монотонная последовательность Хаусдорфа. Для того чтобы из $\left(H, v_{k}\right)$-суммируемости последовательности $\left\{s_{k}\right\}$ следовала сходимость той-же последовательности, необходимо и достаточно, чтобы $\inf v_{k}>0$. 
Дока зате льств о. Докажем достаточность условия. Пусть

$$
t_{n}=\sum_{k=0}^{n}\left(\begin{array}{l}
n \\
k
\end{array}\right) \Delta^{n-k} v_{k} s_{k}
$$

ІІредставим общий элемент метода $(2.5)$ в форме

$$
\left(\begin{array}{l}
n \\
k
\end{array}\right) \Delta^{n-k} v_{k}=\lambda \delta_{n k}+(1-\lambda)\left({ }^{n-1}{ }_{k}\right) \Delta^{n-1-k} \mu_{k} \quad(k=0,1,2, \ldots) .
$$

где пусть при $0<\lambda<1$

$$
\delta_{n k}=\left\{\begin{array}{l}
\frac{v_{n}}{\lambda}, \quad \text { при } k=n \\
0, \text { при } k \neq n .
\end{array}\right.
$$

Последовательность Хаусдорфа $\left\{\mu_{k}\right\}$ определима системой (2.6). Приведем далее равенство (2.5) к виду

$$
t_{n}=\lambda r_{n}+(1-\lambda) \sum_{k=0}^{n-1}\left({ }^{n}{ }_{k}^{1}\right) \Delta^{n-1-k} \tau_{k} r_{k}
$$

где $r_{k}=\frac{v_{k}}{\lambda} s_{k}$

$$
\Delta^{n-1-k} \tau_{k}=\frac{\lambda}{v_{k}} \Delta^{n-1-k} \mu_{k} \quad(k=0,1,2, \ldots) .
$$

Для доказательства абсолютной монотонности последовательности $\left\{\tau_{k}\right\}$ достаточно показать, что последовательность $\left\{\mu_{k}\right\}$ абсолютно монотонна. Последнее очевидно ввиду (2.6), (2.7), абсолютной монотонности последовательности $\left\{v_{k}\right\}$ и того, что $0<\lambda<1$.

Из условия $\lim _{n} t_{n}=s$ и абсолютной монотонности последовательности $\left\{\tau_{k}\right\}$ по теореме 2.3 вытекает, что $\lim r_{n}=s_{0}$, откуда следует равенство $\lim s_{n}=s_{1}$, если учесть определение последовательности $\left\{r_{k}\right\}$ и условие, что $\left\{v_{k}\right\}-$ абсолютно монотонна и $\inf _{k} v_{k}>0$. Этим достаточность условия доказана.

Докажем теперь его необходимость. Пусть при абсолютно монотонной последовательности Хаусдорфа $\left\{v_{k}\right\}$ из сходимости последовательности $t_{n}=\sum_{k=0}^{n}\left(\begin{array}{l}n \\ k\end{array}\right) \Delta^{n-k} v_{k} s_{k}$ вытекает сходимость последовательности $\left\{s_{k}\right\}$. Это означает, что метод $\left(H, \frac{l}{v_{k}}\right)$ сохраняет сходимость, т. е. должен удовлетворять условию

$$
\lim _{n}\left(\begin{array}{c}
n \\
k
\end{array}\right) \Delta^{n-k} \frac{1}{v_{k}}=\alpha_{k} \quad(k=0,1,2, \ldots)
$$

прн сходящемся $\sum_{k=0}^{\infty}\left|\alpha_{k}\right|$. Последнее означает в частности, что последовательность $\left\{a_{k}\right\}$ ограничена. 
Из (2.9), абсолютной монотонности $\left\{v_{k}\right\}$ и ограниченности $\left\{a_{k}\right\}$ вытекает

$$
0 \leqslant \frac{1}{v_{k}} \leqslant M
$$

T. e.

$$
\inf _{k} v_{k}>0
$$

что и требовалось доказать.

Следствие. Если последовательность Хаусдорфа $\left\{v_{k}\right\}$ абсолютно монотонна $u$ inf $v_{k}>0$, тo

$k$

$$
\sup _{n} \sum_{k=0}^{n}\left(\begin{array}{l}
n \\
k
\end{array}\right)\left|\Delta^{n-k} \frac{1}{v_{k}}\right| \neq \infty
$$

Доказательство. Из абсолютной монотонности последовательности Хаусдорфа $\left\{v_{k}\right\}$ и известных теорем (207 и 208 из $\left.\left[{ }^{8}\right]\right)$ вытекает, что метод $\left(H, v_{k}\right)$ сохраняет сходимость. Обозначая множество всех $\left(H, v_{k}\right)$-суммируемых последовательностей символом $\left(H, v_{k}\right)$, можем на основании вышесказанного выписать соотношение $c \subset\left(H, v_{k}\right)^{*}$. Так как по условию inf $v_{k}>0$, то $\left(H, v_{k}\right)^{*} \subseteq c$, что вытекает из теоремы 2.4. Эти результаты и показывают, что метод $\left(H, v_{k}\right)$ суммирует только сходящиеся послеловательности, а по теореме 1.2 это означает, что именно

$$
\sup _{n} \sum_{k=0}^{n}\left(\begin{array}{c}
n \\
k
\end{array}\right)\left|\Delta^{n-k} \frac{1}{v_{k}}\right| \neq \infty \text {. }
$$

Сравнивая нашу теорему 2.4 с теоремой 1.1 Питта и Петерхауса, мы видим, что

1) в теореме 1.1 рассматриваются регулярные методы Хаусдорфа, в нашей же теореме - такне методы Хаусдорфа, 'которые сохраняют сходимость, но у которых последовательности Хаусдорфа абсолютно монотонны;

2) необходимое и достаточное условие теоремы 2.4 проще соответствующего условня в теиреме 1.1 .

Әто показывает, что рассматриваемые теоремы не тождественны, но аналогичны. На основании приведенных доказательств и сравнения результатов Питта и Петерхауса с напими мы приходим к выводу, что для преобразований Мерсера-Хаусдорфа, т. е. преобразований вида (2.1) естественно считать аналогом теоремы 1.1 теорему 2.2. В этом и заключается тесная связь теорем $2.1,2.2$ и 1.1 .

3. Перейдем к доказательству обобщенных теорем Мерсера для двойных последовательностей, т. е. теорем, аналогичных теоремам 2.2, 2.4 и 1.1. При этом будем рассматривать только факторизирующиеся методы Хаусдорфа.

Т е ор ем а 3.1. Пусть $\lambda>0, h \neq \infty,\left\{\mu_{k}\right\} u\left\{v_{l}\right\}$ - абсолютно монотонные последовательности Хаусдорфа, удовлетворяющие условиям $\lim \Delta^{m} \mu_{0}=\lim \Delta^{n} v_{0}=0$. Для того чтобы из равенства

$$
b-\lim _{m, n}\left(\lambda s_{m n}+(1-\lambda) \sum_{k, l=0}^{m-1, n-1}\left({ }_{k}^{m-1}\right)\left({ }^{n-1}\right)^{-1} \Delta^{m-1-k, n-1-l} \mu_{k} v_{l} s_{k l}\right)=h
$$

йтекало равенство

необходимо $и$ достаточно, чтобы $\mu_{0} v_{0}=1$.

$$
b-\lim s_{m, n}=h,
$$


Доказательство теоремы аналогично доказательству теоремы 2.2 (причем вместо 2.1 из [] применим 2.1 из $[$ [] и некоторые результаты из доказательства леммы 1.1 в [5]).

Т е о рем а 3.2. Пусть $\left\{\mu_{k}\right\} u\left\{v_{l}\right\}$ - абсолютно монотонные регулярнысе послејовательности Хаусдорфа. Для того чтобы из ограниченной $\left(H, \mu_{k} v_{l}\right)$-суммируемости последовательности $\left\{s_{k l}\right\}$ следовала ограниченная сходимость последовательности $\left\{s_{k l}\right\}$, необходимо и достаточно, чтобы

$$
\inf _{k} \mu_{k}>0 \quad \text { и } \quad \inf _{l} v_{l}>0 .
$$

Док а з ательство. Во-первых, докажем необходимость условий (3.1). Пусть

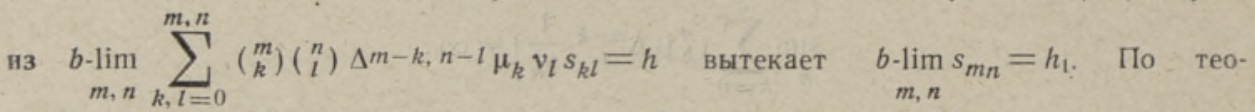
реме 1.3 обратный метод для $\left(H, \mu_{k} v_{l}\right)=\left(H, \mu_{k}\right) \odot\left(H, v_{l}\right)$ имеет вид $\left(H, \frac{1}{\mu_{k}}\right) \odot\left(H, \frac{1}{v_{l}}\right)=\left(H, \frac{1}{\mu_{k} v_{l}}\right)$. Сделанное выше предположение означает, что $\left(H, \frac{1}{\mu_{k} \nu_{l}}\right)$ сохраняет сходимость. По теоремам 1.5 и 1.6 теперь следует, что методы $\left(H, \frac{1}{\mu_{k}}\right)$ и $\left(H, \frac{1}{v_{l}}\right)$ сохраняют сходимость. Дальнейшее доказательство необходимости условий (3.1) совпадает с доказательством необходимости условия в теореме 2.4 .

Докажем достаточность условий $(3.1)$.

Пусть $\left\{\mu_{k}\right\}$ и $\left\{v_{l}\right\}-$ абсолютно монотонные регулярные последовательности Хаусдорфа, удовлетворяющие условиям (3.1); пусть также

$$
b-\lim _{m, n} \sum_{k, l=0}^{m, n}\left(\begin{array}{c}
m \\
k
\end{array}\right)\left(\begin{array}{l}
n \\
l
\end{array}\right) \Delta^{m-k, n-l} \mu_{k} v_{l} s_{k l}=h .
$$

Гогда из следствня теоремы 2.4 вытекает неравенство

$$
\sup _{m, n} \sum_{k, l=0}^{m, n}\left(\begin{array}{l}
m \\
k
\end{array}\right)\left(\begin{array}{l}
n \\
l
\end{array}\right)\left|\Delta^{m-k, n-l} \frac{1}{\mu_{k} v_{l}}\right| \neq \infty .
$$

Поэтому $\left\{s_{k l}\right\}$ может быть только ограниченная и сходящаяся последовательность (см. лемму 1.1 из [7] и теорему 1.7 ).

Этим теорема доказана.

Те орем а 3.3. Пусть $\mathrm{X}(t)$ и $\Psi(t)$ - функции с ограниченным изменением без сингулярной составляющей и удовлетворяющие условиям

$\mathbf{X}(+0)=\mathrm{X}(0)=0, \quad \mathrm{X}(1)=1 \quad$ и $\quad \Psi(+0)=\Psi(0)=0, \quad \Psi(1)=1$.

Для того чтобы из ограниченной сходимости последовательности

$$
t_{m n}=\sum_{k, l=0}^{m, n}\left(\begin{array}{c}
m \\
k
\end{array}\right)\left(\begin{array}{l}
n \\
l
\end{array}\right) \Delta^{m-k, n-l} \mu_{k} v_{l} s_{k l}
$$

где $\mu_{k}=\int_{0}^{1} t^{k} d \mathrm{X}(t) \quad u \quad v_{l}=\int_{0}^{1} t^{l} d \Psi(t)$, вытекала ограниченная сходимость $\left\{s_{\boldsymbol{k}}\right\}$,

необходимо и достаточно, чтобы 


$$
\left.\begin{array}{l}
\inf _{R \omega>0}\left|\int_{0}^{1} t^{\omega} d \mathrm{X}(t)\right|>0 \\
\inf _{R \zeta>0}\left|\int_{0}^{1} t^{\zeta} d \Psi(t)\right|>0 .
\end{array}\right\}
$$

Доказательство. Докажем необходимость условий (3.3), По этим условиям методы $\left(H, \mu_{k}\right)=\left(H, \int_{0}^{1} t^{k} d \mathrm{X}(t)\right)$ и $\left(H, v_{l}\right)=\left(H, \int_{0}^{1} t^{l} d \Psi(t)\right)$ регулярны. Также, по условию, $\left(H, \frac{1}{\mu_{k} v_{l}}\right)$ сохраняет сходимость. Но тогда методы $\left(H, \frac{1}{\mu_{k}}\right)$ и $\left(H, \frac{1}{v_{l}}\right)$ сохраняют сходимость, что вытекает из теорем 1.5 и 1.6. Дальнейший вывод условий (3.3) совпадает с доказательством необходимости условия вида (3.3) (например в теореме $1.1\left[{ }^{3}\right]$ ).

Докажем достаточность условий.

Пусть $b-\lim t_{m n}=h$ и пусть выполнены предположения нашей теоремы относительно методов $\left(H, \mu_{k}\right)$ и $\left(H, v_{l}\right)$. Тогда метод $\left(H, \mu_{\mathbf{k}} v_{l}\right)$ не может суммировать расходящихся последовательностей, т. к. условие теоремы 1.7 не выполнено ввиду леммы 1.1 и условий нашей теоремы. Из последнего вытекает и ограниченность суммируемой последовательности.

Теорема доказана.

\section{Л ИТЕ РА Т У Р А}

1. A d a ms, C. R., On summability of double series. Trans. Anier. Math. Soc., 34, 1932. No. 2, 215-230.

2. Adams, C. R., Hausdorff transformations for double sequences. Bull. Amer. Math. Soc., 39, 1933, 303-312.

3. Pitt, H, R. and Peterhouse, Ph. D., Mercerian theorems. Proc. Cambridge Philos. Soc., 34, 1938, 510-520.

4. Wilansky, A., A necessary and sufficient condition that a summability method be stronger than convergence. Bull. Amer. Math. Soc., 1949, 55, 914-916.

5. С ырмус Т., Об одном обобщении теоремы Мерсера для двойных последовательностей. Уч. зап. Тартуского гос. ун-та, 102, 1961, 156-168.

6. С ырмус Т., О некоторых обобщениях теоремы Мерсера. Уч. зап. Тартуского гос. ун-та, 102, 1961, 169-184.

7. С ырм ус Т., О некоторых обобщениях теоремы Мерсера для двойных последовательностей. Изв. АН ЭССР, сер. физ.-матем. и техн. наук, ХI, 1, 1962, 37-49.

8. Х а рди Г., Расходящиеся ряды, Москва, 1949.

Тартуский государственный университет

Поступила в редакцию

5. V 1961 


\section{OLDISTATUD MERCERI TEOREEMIST}

\section{T. Sõrmus}

\section{Resümee}

Pitt, H. R. ja Peterhouse, Ph. D. [ $\left.{ }^{3}\right]$ tōestasid teoreemi (teoreem 1.1), mis annab tarvilikud ja piisavad tingımused seileks, et jada $\left(H, \mu_{k}\right)$-summeeruvusest järelduks jada koonduvus.

Siin tōestatakse teoreem (teoreem 2.2), mis asendab Pitti ja Peterhouse'i teoreemi Merceri tüüpi teoreemides esinevate menetluste korral. Peale selle näidatakse, et Pitti ja Peterhouse'i teoreemis esinevat tarvilikku ja piisavat tingimust on vōimalik lihtsustada, kui Hausdorffi menetlust määrav jada on totaalselt monotoonne (teoreem 2.4).

Lõpuks antakse ülalmainitud kolmele teoreemile vastavad üldistused kahekordsete jadade tarvis, piirdudes sealjuures faktoriseeruvate Hausdorffi menetlustega (teoreemid $3.1,3.2$ ja 3.3 ).

Tartu Riiklik Olikool

Saabus toimetusse

5. V 1961

\section{UBER DEN VERALLGEMEINERTEN MERCERSCHEN SATZ}

\section{T. Sõrmus}

\section{Zusammenfassung}

Pitt, H. R. und Peterhouse, Ph. D. $\left[^{3}\right]$ haben den Satz bewiesen (Satz 1.1), der eine notwendige und hinreichende Bedingung dazu aufstellt, dass aus der $\left(H, \mu_{k}\right)$-Summierbarkeit einer Folge ihre Konvergenz folgt.

Das Ziel des Artikels ist, zu zeigen, dass man die Pittsche und Peterhousesche notwendige und hinreichende Bedingung für die folgenden Verfahren noch vereinfachen kann:

1) das Verfahren, das durch die Transformation

$$
t_{n}=\lambda_{s n}+(1-\lambda) \sum_{k=0}^{n-1}\left({ }_{k}^{n-1}\right) \Delta^{n-1-k} \mu_{k}{ }^{s}
$$

bestimmt ist;

2) das mit Hilfe der total-monotonen Folge $\left\{\mu_{k}\right\}$ definierte $\left(H, \mu_{k}\right)$-Verfahren [die Sätze 2.2 und 2.4].

Schliesslich werden die drei Sätze für den Fall der Doppelfolgen verallgemeinert (Sätze 3.1, 3.2 und 3.3). 